OPEN ACCESS

Edited by:

Ramon Diaz-Arrastia

University of Pennsylvania,

United States

Reviewed by:

John K. Yue,

University of California, San Francisco,

United States

Denes V. Agoston,

Uniformed Services University of the Health Sciences, United States

Kimbra Kenney,

Uniformed Services University of the Health Sciences, United States

*Correspondence:

Torgeir Hellstrøm torgeir.hellstrom@ous-hf.no

Specialty section: This article was submitted to Neurotrauma

a section of the journal

Frontiers in Neurology

Received: 02 July 2021 Accepted: 21 January 2022 Published: 16 February 2022

Citation:

Hellstrøm T, Andelic N, Holthe ØØ, Helseth E, Server A, Eiklid K and Sigurdardottir S (2022) APOE-\&4 Is Associated With Reduced Verbal Memory Performance and Higher Emotional, Cognitive, and Everyday Executive Function Symptoms Two Months After Mild Traumatic Brain Injury. Front. Neurol. 13:735206 doi: 10.3389/fneur.2022.735206

\section{APOE- $\varepsilon 4$ Is Associated With Reduced Verbal Memory Performance and Higher Emotional, Cognitive, and Everyday Executive Function Symptoms Two Months After Mild Traumatic Brain Injury}

\author{
Torgeir Hellstrøm ${ }^{1 *}$, Nada Andelic ${ }^{1,2}$, Øyvor Øistensen Holthe ${ }^{1}$, Eirik Helseth ${ }^{3,4}$, \\ Andres Server ${ }^{5}$, Kristin Eiklid ${ }^{6}$ and Solrun Sigurdardottir ${ }^{7}$ \\ ${ }^{1}$ Department of Physical Medicine and Rehabilitation, Oslo University Hospital, Oslo, Norway, ${ }^{2}$ Research Center for \\ Habilitation and Rehabilitation Models and Services (CHARM), Institute of Health and Society, University of Oslo, Oslo, \\ Norway, ${ }^{3}$ Institute of Clinical Medicine, Faculty of Medicine, University of Oslo, Oslo, Norway, ${ }^{4}$ Department of Neurosurgery, \\ Oslo University Hospital, Oslo, Norway, ${ }^{5}$ Section of Neuroradiology, Department of Radiology and Nuclear Medicine, Oslo \\ University Hospital, Oslo, Norway, ${ }^{6}$ Department of Medical Genetic, Oslo University Hospital, Oslo, Norway, ${ }^{7}$ Centre for Rare \\ Disorders, Oslo University Hospital, Oslo, Norway
}

Background: Substantial variance exists in outcomes after mild traumatic brain injury (MTBI), and these differences are not fully explained by injury characteristics or severity. Genetic factors are likely to play a role in this variance.

Objectives: The aim of this study was to examine associations between the apolipoprotein (APOE)- $\varepsilon 4$ allele and memory measures at two months post-MTBI and to evaluate whether subjective cognitive and affective symptoms were associated with APOE- $\varepsilon 4$ status. Based on previous research, it was hypothesized that APOE- $\varepsilon 4$ carriers would show poorer verbal memory performance compared to APOE- $\varepsilon 4$ non-carriers.

Methods: Neuropsychological data at two months post-injury and blood samples that could be used to assess APOE genotype were available for 134 patients with MTBI (mean age 39.2 years, 62\% males, 37\% APOE- 44 carriers). All patients underwent computed tomography at hospital admission and magnetic resonance imaging four weeks post-injury.

Results: The APOE- $\varepsilon 4$ + status was associated with decreased immediate memory recall $(p=0.036 ; \beta=-0.10,95 \% \mathrm{Cl}[-0.19,-0.01])$. Emotional, cognitive, and everyday executive function symptoms at two months post-injury were significantly higher in APOE- $\varepsilon 4$ carriers compared to non-carriers.

Conclusion: The APOE- $\varepsilon 4+$ allele has a negative effect on verbal memory and symptom burden two months after MTBI.

Keywords: mild traumatic brain injury, APOE, learning, memory, affective symptoms 


\section{INTRODUCTION}

Individuals after a mild traumatic brain injury (MTBI) can display neuropsychological functioning ranging from normal to poor cognitive recovery, and subjective complaints can differ widely, from no symptoms to prolonged cognitive, emotional, and somatic symptoms (1). Several factors influence neurocognitive and functional outcomes, including demographic (e.g., age at injury, education), psychosocial (e.g., socioeconomic status, family status), premorbid functioning (e.g., intellectual ability, employment), and injury-related variables (e.g., severity, external cause of injury) (2-6). Over the last decade, an increasing number of studies have suggested that genetic polymorphisms, particularly the apolipoprotein (APOE) genotype, may play a pivotal role in the recovery process and help explain the heterogeneity in neurocognitive outcomes following MTBI (711). However, despite the growth in genetic and genomic research, the influence of genetic factors on neuropsychological performance, functional outcome, and neuroimaging findings following MTBI has yet to be fully investigated. It would be clinically meaningful to better understand heterogeneity in neurocognitive outcomes and the factors that contribute to increased risk of decline in memory over time in response to MTBI, as these deficits may reflect underlying brain dysfunction (12-14).

Findings from previous studies have shown that the APOE genotype is predictive of important outcomes after a TBI, including memory and processing speed, verbal episodic memory performance, level of global functioning, and mortality $(8,15-$ $20)$. The product of the APOE gene serves many functions within the central nervous system, including lipid transportation and clearance, as well as maintenance of neuronal integrity and synapto-dendritic connections $(21,22)$. There are three common allelic variations of the $A P O E$ gene, $\varepsilon 2, \varepsilon 3$, and $\varepsilon 4$, and these variants differ notably in their capacity for stimulating neurite outgrowth following a central nervous system insult $(22,23)$. Compared to the $\varepsilon 2$ and $\varepsilon 3$ alleles, the $\varepsilon 4$ allele is detrimental in this process, as it inhibits neurite outgrowth, disrupts neuronal cytoskeleton, and magnifies amyloid beta accumulation $(22,23)$. Furthermore, APOE- $\varepsilon 4$ is a well-known genetic risk factor for Alzheimer's disease and markedly exacerbates taumediated neurodegeneration (24), which can negatively affect cognitive functioning (22). Additionally, within the context of TBI, the $\varepsilon 4$ allele has been linked with increased likelihood of cerebrovascular pathology (25), larger hematoma volume (26), and the presence of cerebral amyloid angiopathy (27).

The Glasgow Outcome Scale Extended (GOSE) is often used to measure the relationship between the APOE- 84 allele and global patient outcomes following TBI. In a meta-analysis by Zhou et al. (15), APOE- $\varepsilon 4$ carriage was associated with a higher risk of poor outcomes six months post-TBI. Another metaanalysis, by Zeng et al. (16), showed that the APOE- $\varepsilon 4$ allele was associated with lower odds of a good prognosis, with a slightly larger effect size if only severe TBI was included. Further, a meta-analysis by McFayden et al. (17) demonstrated higher odds of a favorable functional outcome following TBI in APOE$\varepsilon 4$ non-carriers compared with APOE- $\varepsilon 4$ carriers and individual homozygous for this allele. In a study of college athletes who sustained MTBI, Merritt et al. reported that APOE- $\varepsilon 4$ carriers had significantly worse self-reported symptomatology at three months after injury, mostly within the physical and cognitive domains (28). However, in line with a study of professional fighters (29) and athletes (30), no clear effect of the APOE- 84 allele on neuropsychological functioning was established (17). Several studies have reported a negative effect of the APOE$\varepsilon 4$ allele on memory $(29,30)$. For example, one study found that the APOE- $\varepsilon 4$ allele may confer an increased risk of verbal memory impairment at six months after MTBI (7). Another study showed that the $\varepsilon 4$ allele was associated with worse memory and processing speed as well as overall cognitive impairment among military veterans (8). Notably, however, a meta-analysis by Padgett et al. reported no significant differences in general cognitive or memory functions after TBI (31). A systematic review by Lawrence et al. (32) concluded that the effect of APOE- 84 on TBI outcome was non-contributory in 14 studies (58.3\%), hazardous in $9(37.5 \%)$, and protective in $1(4.2 \%)$. Most of the studies reported on fewer than 200 patients. Taken together, meta-analyses and systematic reviews have reported different effects of the APOE- $\varepsilon 4$ on TBI outcomes in studies describing outcomes for diverse populations of patients with mild, moderate, or severe TBI, as well as patients with different characteristics (pediatric, adolescent, adult, older adult) and in different settings in which the TBI was sustained (civilian, sportsrelated, military).

In the present study, we evaluated a homogenous sample of adult patients with MTBI. All patients were hospitalized due to the injury and followed up at two months post-injury. The aim was to examine the associations between the APOE- $\varepsilon 4$ allele and five verbal memory measures at two months after injury. Further, we assessed whether subjective cognitive and affective symptoms at two months post-injury were associated with APOE- $\varepsilon 4$ status, as well as group differences between $\varepsilon 4(+)$ carriers and noncarriers of $\varepsilon 4(-)$ determined using neuroimaging with the use of acute computed tomography (CT) and magnetic resonance imaging (MRI) at four weeks post-injury. Based on previous literature, we hypothesized that APOE- $\varepsilon 4$ would have a negative impact on verbal memory and that APOE- 84 carriers with MTBI would experience more cognitive and affective symptoms compared to APOE- $\varepsilon 4$ non-carriers.

\section{MATERIALS AND METHODS}

\section{Participants and Procedure}

Patients with an acute MTBI who were admitted to the Oslo University Hospital, Oslo, Norway, between September 2011 and September 2013 were included in a prospective cohort study. Patients aged $16-65$ years with a recent $(<24 \mathrm{~h})$ history of head trauma (hospitalization with ICD-10 diagnosis S06.0S06.9) resulting in a loss of consciousness (LOC) $<30 \mathrm{~min}$, posttraumatic amnesia $(\mathrm{PTA})<24 \mathrm{~h}$, and a Glasgow Coma Scale (GCS) score between 13 and 15 were included. MTBI was defined using criteria from the American Congress of Rehabilitation Medicine (33). Exclusion criteria were confirmed diagnosis of severe mental illness in medical records (e.g., schizophrenia 
or bipolar disorder), progressive neurologic disease, ICD-10 diagnosis of substance dependence, contraindications for MRI, and lack of Norwegian language skills.

\section{Biospecimen and Genotyping Procedures}

The variants in exon four in the APOE gene (GenBank: NM_000041) - that is, c.388T $>$ C and c.526C > T-were analyzed by polymerase chain reaction and Sanger sequencing in DNA extracted from peripheral leukocytes. Primers were designed using Primer3Plus (Bioin-formatics, Arlington, VA, USA) (34) and the polymerase chain reaction products were purified and Sanger sequenced using an ABI 3730xl DNA analyzer and an ABI BigDye terminator cycle-sequencing kit v3.1 (Thermo Fisher Scientific, Waltham, MA, USA). Sequences were analyzed with the DNA Sequencing Analysis software program (version 5.1; Applied Biosystems, Foster City, CA, USA) and the SeqScape software program (version 2.7; Thermo Fisher Scientific).

\section{Patient Groups: APOE- $\varepsilon 4(+)$ and APOE- $\varepsilon 4(-)$}

For the current analyses, patients with available neuropsychological data at two months post-injury and APOE blood samples were included. Of the 176 participants included at 2 months (35), complete data were available for 138 , but 4 patients were excluded due to lack of motivation (see below). Fifty of the patients included (37\%) were APOE- $\varepsilon 4(+)$ carriers and 84 (63\%) were APOE- $84(-)$ non-carriers.

Information regarding age, sex, and education level was obtained from a clinical interview. The GCS (36) was used to assess the conscious state, and total scores on this scale can be between 3 (showing no response) and 15 (alert and welloriented). The presence and duration of PTA and LOC were determined based on medical records and classified into no PTA vs. yes/unknown and no LOC vs. yes/unknown. External cause of injury was obtained from medical records and classified as traffic accidents, falls, violence, or other. The CT taken at admission to hospital (CT acute) on clinical indication was used to assess intracranial injuries.

\section{MRI Data Acquisition and Analysis}

3T MRI (GE Signa HDxt, GE Medical Systems, Milwaukee, WI, USA) data was obtained four weeks post-injury using two different head coils (Head/Neck/Spine [HNS] and 8HRBRAIN). MRI scans were performed using a 3T whole-body MRI system. The protocol included a 3D Fast Spoiled Gradient Echo (FSPGR) T1-weighted sequence used for morphometric assessments (repetition time msec/echo time msec/inversion time msec, 7,8744/2.96/450; flip angle, $12^{\circ}$; and spatial resolution, $1 \times 1.3 \times 1.2 \mathrm{~mm}$ ). Acquisition parameters were optimized for increased gray/white matter contrast. In addition, a T2weighted sequence and a T2 susceptibility-weighted angiography (SWAN) sequence were performed to depict hemorrhagic or other lesions. No major scanner upgrade occurred during the study period. MRI scans were evaluated for gross pathologies by a neuroradiologist.

\section{Neuropsychological Assessment}

Participants were assessed by one neuropsychologist without prior knowledge of the participants' $A P O E$ genotypes. In this study, the raw scores of five sub-tests of the California Verbal Learning Test-II (CVLT-II) (37) were of interest to assess learning and memory: recall of 16 words presented over five trials (Immediate Recall Trials 1-5), two short-delay recalls (ShortDelay Free Recall and Short-Delay Cued Recall), and two delay recalls of the word list after 20 min (Long-Delay Free Recall and Long-Delay Cued Recall). The CVLT-II is recommended for use in the TBI population and is reported to have excellent reliability and validity (38). The CVLT-II was also used in a similar study by Yue et al. (7), making it possible to compare our results with their findings.

The Rey Fifteen-Item Test, which is used to assess symptom validity, was used to measure lack of motivation. In this test, the participant is shown 15 items for $10 \mathrm{~s}$ and is then requested to draw what they recall. In this study, a lack of motivation was defined as a score of $\leq 9$. Four patients were excluded due to low scores.

\section{Self-Reported Outcomes}

The Behavioral Rating Inventory of Executive Function-Adult Version (BRIEF-A) (39) consists of 75 items that assess nine aspects of executive functioning in everyday life, with responses scored from 1 (never) to 3 (often). The BRIEF-A consists of a composite index score, the Global Executive Composite (GEC), and two sub-index scores; the Behavioral Regulation Index (BRI) and the Metacognition Index (MI). Raw scores are transformed into age-corrected T-scores.

The Rivermead Post-Concussion Symptoms Questionnaire (RPQ) assesses frequently occurring symptoms in the cognitive, emotional, and somatic domains (40). Individuals were asked to rate symptoms over the past $24 \mathrm{~h}$ on a scale from 0 (not experienced at all) to 4 (a severe problem). The items from each sub-scale were summed to a total score, with ratings of 1 excluded. The sub-scale scores (RPQ somatic, emotional and cognitive) and the total RPQ score were used in this study.

The Hospital Anxiety and Depression Scale (HADS) (41) has two subscales (Anxiety and Depression), each consisting of seven items rated on a 4-point scale from 0 (no symptom) to 3 (severe symptom). The validity and reliability of the HADS has been established in patients with TBI $(42,43)$. Both the sub-scales scores (range $0-21$ ) and the total HADS score (range $0-42$ ) were used in the analyses.

\section{Functional Outcome}

The GOSE measures global function, including independence; work, social, and leisure activities; and participation in social life (44). It is an 8-point ordinal scale reflecting good recovery $(7,8)$, moderate $(5,6)$ and severe $(3,4)$ disability, vegetative state $(2)$, and death (1).

\section{Statistical Analysis}

Descriptive statistical analyses were performed using SPSS for Windows, version 26 (SPSS Inc., Chicago, IL, USA). Sample characteristics are presented as the group mean and 
TABLE 1 | Demographics and injury-related characteristics stratified by the APOE- 44 status groups.

\begin{tabular}{|c|c|c|c|c|}
\hline Variables & $\begin{array}{l}\text { MTBI sample } \\
\qquad(N=134)\end{array}$ & $\begin{array}{c}\text { APOE } \\
\varepsilon 4(-) \\
(N=84)\end{array}$ & $\begin{array}{c}\text { APOE } \\
\varepsilon 4(+) \\
(N=50)\end{array}$ & $p$-value \\
\hline Age (years) & $39.2(14.4)$ & 40.7 (14.3) & $37.0(14.5)$ & 0.16 \\
\hline $\begin{array}{l}\text { Gender } \\
\text { - Male } \\
\text { - Female }\end{array}$ & $\begin{array}{l}83(62 \%) \\
51(38 \%)\end{array}$ & $\begin{array}{l}51(61 \%) \\
33(39 \%)\end{array}$ & $\begin{array}{l}32 \text { (64\%) } \\
18 \text { (36\%) }\end{array}$ & 0.71 \\
\hline $\begin{array}{l}\text { Education } \\
\text { - Less than } 12 \text { years } \\
\text { - Over } 12 \text { years }\end{array}$ & $\begin{array}{l}63(47 \%) \\
71(53 \%)\end{array}$ & $\begin{array}{l}38 \text { (45\%) } \\
46(55 \%)\end{array}$ & $\begin{array}{l}25 \text { (50\%) } \\
25(50 \%)\end{array}$ & 0.59 \\
\hline $\begin{array}{l}\text { Mechanism of injury } \\
\text { - Traffic accidents } \\
\text { - Falls } \\
\text { - Violence } \\
\text { - Other }\end{array}$ & $\begin{array}{l}51(38 \%) \\
52(39 \%) \\
17(13 \%) \\
14(10 \%)\end{array}$ & $\begin{array}{c}31(37 \%) \\
36(43 \%) \\
10(12 \%) \\
7(8 \%)\end{array}$ & $\begin{array}{l}20(40 \%) \\
16(32 \%) \\
7(14 \%) \\
7(14 \%)\end{array}$ & 0.55 \\
\hline $\begin{array}{l}\text { GCS score } \\
-13-14 \\
-15\end{array}$ & $\begin{array}{l}38(28 \%) \\
96(72 \%)\end{array}$ & $\begin{array}{l}26(31 \%) \\
58(69 \%)\end{array}$ & $\begin{array}{l}12(24 \%) \\
38(76 \%)\end{array}$ & 0.39 \\
\hline $\begin{array}{l}\text { LOC } \\
\text { - No } \\
\text { - Yes/unknown }\end{array}$ & $\begin{array}{c}26(19 \%) \\
108(81 \%)\end{array}$ & $\begin{array}{l}19(23 \%) \\
65(77 \%)\end{array}$ & $\begin{array}{c}7(14 \%) \\
43(86 \%)\end{array}$ & 0.22 \\
\hline $\begin{array}{l}\text { PTA (n, \%) } \\
\text { - No amnesia } \\
\text { - Yes/unknown }\end{array}$ & $\begin{array}{c}13(10 \%) \\
121(90 \%)\end{array}$ & $\begin{array}{l}10(12 \%) \\
74(88 \%)\end{array}$ & $\begin{array}{c}3(6 \%) \\
47(94 \%)\end{array}$ & 0.26 \\
\hline Length of hospital stay (days) & 2.5 (3.3) & $2,29(2,32)$ & $2,88(4,52)$ & 0.39 \\
\hline $\begin{array}{l}\text { MRI and CT pathology } \\
\text { - No } \\
\text { - Yes }\end{array}$ & $\begin{array}{l}68(51 \%) \\
66(49 \%)\end{array}$ & $\begin{array}{l}43(51 \%) \\
41(49 \%)\end{array}$ & $\begin{array}{l}25(50 \%) \\
25(50 \%)\end{array}$ & 0.89 \\
\hline $\begin{array}{l}\text { Site of injury on MRI }(n=131) \\
\text { - Frontal } \\
\text { - Temporal } \\
\text { - Frontotemporal } \\
\text { - Parietal } \\
\text { - Occipital }\end{array}$ & $\begin{array}{c}29(22 \%) \\
23(17 \%) \\
11(8 \%) \\
13(10 \%) \\
7(5 \%)\end{array}$ & $\begin{array}{c}14(11 \%) \\
16(12 \%) \\
5(4 \%) \\
9(7 \%) \\
6(5 \%)\end{array}$ & $\begin{array}{c}15(11 \%) \\
7(5 \%) \\
6(5 \%) \\
4(3 \%) \\
1(0.8 \%)\end{array}$ & $\begin{array}{l}0.09 \\
0.40 \\
0.33 \\
0.77 \\
0.25\end{array}$ \\
\hline
\end{tabular}

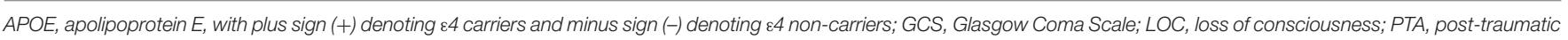
amnesia; CT, computed tomography; MRl, magnetic resonance imaging.

p-values: T-test for continuous variables; Chi square and Fischer's exact test for categorical variables. Data are presented as means (SD) or frequencies (n, \%).

standard deviation (SD). Differences between patient groups on continuous variables were tested using Student's $t$-test. The Chi-square test was used to detect group differences in categorical variables. Statistical power was calculated by the program $G^{*}$ power (45). The sample size of 134 would give a statistical power of 0.88 at $\alpha$ level of 0.05 , with medium effect size $\left(F^{2}=0.15\right)$ and nine predictors.

Tests of normality and skewness indicated that the CVLTII, age at the time of the two-month assessment, and length of stay (LOS) at the emergency hospital deviated from a normal distribution. Logarithmic transformations of these variables were therefore used in the regression analyses, with lower scores on the CVLT-II reflecting better performance, lower scores on age indicating lower age, and lower scores in LOS indicating fewer days at the emergency hospital.

Hierarchical multiple regressions were conducted to assess the relationship between the five CVLT-II outcome measures and the APOE- $\varepsilon 4$ genotype, using a bootstrap with 1,000 replications. The regression analyses are presented as $\beta$ coefficients, with $95 \%$ confidence intervals ( $95 \% \mathrm{CI}$ ) for each predictor, $p$-values, explained variance $\left(R^{2}\right)$, and the $\Delta R^{2}$. For the first step, the predictor variables of age at the two-month assessment, education $(0=$ over 12 years, $1=12$ years or less $)$, gender $(0=$ male, $1=$ female $)$, employment $(0=$ employed, $1=$ unemployed $),$ LOS at the emergency hospital, LOC $(0=$ no, $1=$ yes $)$, PTA $(0=$ no amnesia, $1=$ amnesia $)$, and brain CT and MRI brain scans $(0=$ uncomplicated MTBI without CT/MRI findings, 1 = complicated MTBI with CT/MRI findings) were included. Genotype was added in the second step $[0=$ APOE$\varepsilon 4(+), 1=$ APOE- $\varepsilon 4(-)]$. Findings with a two-tailed $p$-value of $<0.05$ were considered statistically significant.

\section{RESULTS}

\section{Patients and Injury Characteristics}

The overall sample ( $n=134$ MTBI patients) was predominantly male $(62 \%)$ and had a mean age of 39.2 years (SD 14.4). When stratified by APOE- $\varepsilon 4$ status, the $\varepsilon 4(+)$ and the $\varepsilon 4(-)$ groups did not differ significantly in any demographic or clinical variables (Table 1). The majority of the injuries occurred due to falls (39\%), 
followed by traffic injuries (38\%). The majority of the patients had a GCS score of $15(72 \%)$, and there were 68 uncomplicated and 66 complicated MTBIs. None of the patients had undergone intracranial surgery. Psychiatric or medical comorbidities were extracted and checked in medical records; 11 patients (8\%) had anxiety, 11 patients (8\%) had depression, $10 \%$ were diagnosed with headaches and six patients (4\%) had cardiac diseases. Twenty percent of the 30 patients with comorbidity in our study had two or more comorbidities. Univariate regression analyses did not reveal any significant associations between the abovementioned comorbidities with APOE- $\varepsilon 4$ status or outcome measures (CVLT-II). Hospital length of stay ranged from 1 to 31 days (SD 3.3) and there were no significant difference between the two APOE- $\varepsilon 4$ groups $(p=0.39)$. A small proportion of patients received rehabilitation (6\%) and physiotherapy (10\%) during the two months post injury. Thirty-six patients $(27 \%)$ with persistent symptoms were offered clinical assessments by an outpatient rehabilitation team after the two months follow-up at the Oslo University hospital.

\section{APOE- $\varepsilon 4$ and Memory Outcomes at Two Months Post-injury}

The results of the multiple regressions are presented in Table 2 and show the predictors' standardized coefficients $(\beta)$, their $95 \%$ CIs, and statistically significant effects. The APOE- $\varepsilon 4$ status was significantly associated with the Immediate Recall Trials 1-5 $(p=0.036)$ and improved the model in the second step $\left(\Delta \mathrm{R}^{2}=+0.023\right)$. The APOE- $\varepsilon 4(+)$ genotype was associated with a decrease in immediate memory recall $(\beta=-0.10,95 \% \mathrm{CI}$ $[-0.19,-0.01])$. Lower age $(\beta=0.52,95 \%$ CI $[0.22,0.82])$ and more education ( $\beta=0.24,95 \%$ CI $[0.13,0.34])$ were strongly associated with increased immediate recall $(p$-values $<0.001)$. No other variables emerged as significant predictors.

For the Short-Delay Free Recall, a near-significant effect of APOE- $\varepsilon 4$ status was found $(p=0.085)$ and improved the model $\left(\Delta \mathrm{R}^{2}=+0.020\right)$. Lower age $(\beta=0.34,95 \%$ CI $[0.05,0.61])$ and more education $(\beta=0.20,95 \%$ CI $[0.10,0.29])$ were associated with better performance on the Short-Delay Free Recall ( $p=0.025$ and $p<0.001$, respectively). No other variables emerged as significant predictors. For the other three CVLT-II models, only age and education emerged as significant predictors.

\section{APOE- $\varepsilon 4$, Self-Reported Outcomes, and Global Functioning at Two Months Post-injury}

The APOE- $44(+)$ genotype was generally associated with higher symptom pressure (see Table 3). Specifically, significantly higher scores were observed in the APOE- $\varepsilon 4(+)$ group on dimensions of post-concussive symptoms, as represented by emotional and cognitive symptoms (RPQ) and the Global Executive Composite and Metacognition and Behavioral Regulation indices of the BRIEF-A. There were no other significant differences between the two groups in anxiety or depression. Moreover, the two groups reported equal function on the GOSE.
TABLE 2 | Multivariable regression analyses of APOE- $\varepsilon 4$ genotype and verbal memory subscales (California Verbal Learning Test-II) at two months post-injury.

\begin{tabular}{|c|c|c|}
\hline Variable & $\beta(95 \% \mathrm{Cl})$ & $p$-value \\
\hline Immediate Recall Trial 1-5 & $\begin{array}{c}\left(R^{2}=0.458\right. \\
\left.\Delta R^{2}=+0.023\right)\end{array}$ & \\
\hline APOE- $\varepsilon 4(+)$ & $-0.10[-0.19,-0.01]$ & 0.036 \\
\hline Age (years) & $0.52[0.22,0.82]$ & $<0.001$ \\
\hline Education (over 12 years) & $0.24[0.13,0.34]$ & $<0.001$ \\
\hline Gender (male) & $-0.04[-0.15,0.07]$ & 0.513 \\
\hline Employment (yes) & $-0.05[-0.17,0.09]$ & 0.532 \\
\hline Emergency hospital stay & $-0.15[-0.36,0.02]$ & 0.119 \\
\hline LOC (yes/unknown) & $-0.02[-0.15,0.12]$ & 0.798 \\
\hline PTA (yes/unknown) & $0.06[-0.13,0.29]$ & 0.541 \\
\hline CT/MRI pathology (yes) & $-0.03[-0.14,0.09]$ & 0.666 \\
\hline Short-Delay Free Recall & $\begin{array}{c}\left(R^{2}=0.383\right. \\
\left.\Delta R^{2}=+0.020\right)\end{array}$ & \\
\hline APOE- $\varepsilon 4(+)$ & $-0.09[-0.18,0.01]$ & 0.085 \\
\hline Age (years) & $0.34[0.05,0.61]$ & 0.025 \\
\hline Education (over 12 years) & $0.20[0.10,0.29]$ & $<0.001$ \\
\hline Gender (male) & $0.02[-0.07,0.12]$ & 0.664 \\
\hline Employment (yes) & $-0.06[-0.20,0.07]$ & 0.399 \\
\hline Emergency hospital stay & $-0.08[-0.23,0.07]$ & 0.282 \\
\hline LOC (yes/unknown) & $0.05[-0.09,0.19]$ & 0.450 \\
\hline PTA (yes/unknown) & $0.06[-0.16,0.26]$ & 0.568 \\
\hline CT/MRI pathology (yes) & $0.04[-0.07,0.15]$ & 0.455 \\
\hline Short-Delay Cued Recall & $\begin{array}{c}\left(R^{2}=0.398\right. \\
\left.\Delta R^{2}=+0.007\right)\end{array}$ & \\
\hline APOE- $\varepsilon 4(+)$ & $-0.05[-0.16,0.04]$ & 0.336 \\
\hline Age (years) & $0.34[0.07,0.67]$ & 0.029 \\
\hline Education (over 12 years) & $0.21[0.10,0.31]$ & $<0.001$ \\
\hline Gender (male) & $-0.03[-0.13,0.08]$ & 0.547 \\
\hline Employment (yes) & $-0.01[-0.15,0.14]$ & 0.976 \\
\hline Emergency hospital stay & $-0.16[-0.38,0.01]$ & 0.101 \\
\hline LOC (yes/unknown) & $0.02[-0.11,0.17]$ & 0.749 \\
\hline PTA (yes/unknown) & $0.01[-0.19,0.20]$ & 0.914 \\
\hline CT/MRI pathology (yes) & $0.05[-0.05,0.16]$ & 0.370 \\
\hline Long-Delay Free Recall & $\begin{array}{c}\left(R^{2}=0.407\right. \\
\left.\Delta R^{2}=+0.015\right)\end{array}$ & \\
\hline APOE- $\varepsilon 4(+)$ & $-0.08[-0.17,0.02]$ & 0.119 \\
\hline Age (years) & $0.40[0.09,0.71] 0.20$ & 0.016 \\
\hline Education (over 12 years) & {$[0.10,0.30]-0.03$} & 0.001 \\
\hline Gender (male) & {$[-0.13,0.07]-0.02$} & 0.525 \\
\hline Employment (yes) & {$[-0.17,0.13]-0.11$} & 0.783 \\
\hline Emergency hospital stay & {$[-0.29,0.06] 0.07$} & 0.235 \\
\hline LOC (yes/unknown) & {$[-0.07,0.21] 0.10$} & 0.364 \\
\hline PTA (yes/unknown) & {$[-0.09,0.29] 0.01$} & 0.295 \\
\hline CT/MRI pathology (yes) & {$[-0.10,0.12]$} & 0.892 \\
\hline Long-Delay Cued Recall & $\begin{array}{c}\left(R^{2}=0.429 ;\right. \\
\left.\Delta R^{2}=+0.012\right)\end{array}$ & \\
\hline APOE- $\varepsilon 4(+)$ & $-0.07[-0.18,0.03]$ & 0.165 \\
\hline Age (years) & $0.45[0.16,0.76] 0.21$ & 0.001 \\
\hline Education (over 12 years) & {$[0.11,0.32]-0.01$} & 0.003 \\
\hline Gender (male) & {$[-0.11,0.10] 0.01$} & 0.866 \\
\hline Employment (yes) & {$[-0.15,0.15]-0.13$} & 0.960 \\
\hline Emergency hospital stay & {$[-0.29,0.03] 0.07$} & 0.153 \\
\hline LOC (yes/unknown) & {$[-0.08,0.23] 0.06$} & 0.377 \\
\hline PTA (yes/unknown) & {$[-0.14,0.24] 0.03$} & 0.512 \\
\hline CT/MRI pathology (yes) & {$[-0.08,0.14]$} & 0.560 \\
\hline
\end{tabular}

APOE, apolipoprotein E; CVLT-II, California Verbal Learning Test-2nd edition; $\beta$, standardized effect size; Cl, confidence interval; LOC, loss of consciousness; PTA, post-traumatic amnesia; CT, computed tomography; MRI, magnetic resonance imaging. Bootstrapped regression analyses were used to assess the association of APOE $\varepsilon 4$ genotype on memory performance. 
TABLE 3 | Self-reported outcome measures according to APOE- $\varepsilon 4$ status group.

\begin{tabular}{|c|c|c|c|c|}
\hline $\begin{array}{l}\text { Variables } \\
2 \text { months post-injury }\end{array}$ & $\begin{array}{l}\text { Overall } \\
(N=134)\end{array}$ & $\begin{array}{l}\text { APOE- } \varepsilon 4(-) \\
(N=84)\end{array}$ & $\begin{array}{l}\text { APOE- } \varepsilon 4(+) \\
\quad(N=50)\end{array}$ & $p$-value \\
\hline RPQ-total, mean (SD) & $11.5(12.5)$ & $10.2(12.4)$ & $13.6(12.6)$ & 0.12 \\
\hline RPQ-somatic, mean (SD) & $5.3(6.0)$ & $5.2(6.3)$ & $5.4(5.6)$ & 0.83 \\
\hline RPQ-emotional, mean (SD) & $2.9(4.3)$ & $2.3(4.0)$ & $4.0(4.7)$ & 0.02 \\
\hline RPQ-cognitive, mean (SD) & $3.3(3.7)$ & $2.7(3.4)$ & $4.2(3.9)$ & 0.02 \\
\hline BRIEF-A Global Executive Composite T-score & $48.9(11.3)$ & $47.2(11.5)$ & $51.6(10.5)$ & 0.03 \\
\hline BRIEF-A Metacognition index T-score & $50.0(11.4)$ & $48.3(11.7)$ & $52.9(10.5)$ & 0.02 \\
\hline BRIEF-A Behavioral Regulation Index T-score & $47.8(10.5)$ & $46.4(10.2)$ & $50.0(10.7)$ & 0.05 \\
\hline HAD-total, mean (SD) & $7.51(6.39)$ & $7.30(6,56)$ & $7.88(6,15)$ & 0.61 \\
\hline HAD-anxiety, mean (SD) & $4.71(3.83)$ & $4.55(3.81)$ & $4.98(3.88)$ & 0.53 \\
\hline HAD-depression, mean (SD) & $2.81(3.23)$ & $2.75(3.52)$ & $2.90(2.70)$ & 0.78 \\
\hline GOSE-total, mean (SD) & $6.80(0.91)$ & $6.88(0.92)$ & $6.68(0.89)$ & 0.22 \\
\hline
\end{tabular}

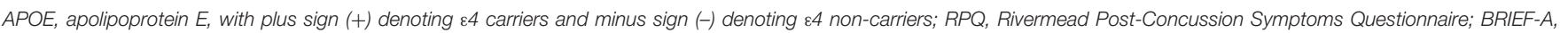
Behavioral Rating Inventory of Executive Function-Adult Version; HADS, Hospital Anxiety and Depression Scale; GOSE, Glasgow Outcome Scale Extended.

\section{APOE- $\varepsilon 4$ and Acute Findings on Brain Scans}

Significantly more epidural hematomas on the acute CT were observed in the APOE- $\varepsilon 4(+)$ group (see Supplementary Table 1). On the MRI at four weeks after MTBI (see Supplementary Table 2), there were no significant differences in type of intracranial injury between the two groups. Diffuse axial injuries (DAI) in the APOE- $\varepsilon 4(+)$ group were more frequent, but this difference was not significant $(p=0.07)$.

\section{DISCUSSION}

The primary aim of the present study was to examine the association between the $\varepsilon 4$ allele of the APOE gene and verbal memory two months after MTBI in a civilian population. Consistent with our hypothesis, APOE- $\varepsilon 4(+)$ was associated with decreased immediate memory recall at two months post-injury. In general, age and education had significant impacts on all five subtests of verbal memory (CVLT-II). Interestingly, in this study, the injury characteristics were not associated with verbal memory performance. Thus, memory deficits in MTBI do not seem to occur solely based on age and education or injury severity. Our study confirms the finding of a study by Yue et al. (7) that APOE$\varepsilon 4$ may confer an increased risk of impairment of verbal memory in the first months after injury. Recent studies on cognitive functions in patients with MTBI have found that cognitive deficits were associated with APOE- $\varepsilon 4$ status in this patient group $(7,8,20)$. In addition, in a study of athletes with concussions, Merritt et al. (30) demonstrated greater neurocognitive variability in APOE- $\varepsilon 4$ carriers $(+)$ compared to non-carriers. Such findings are in line with those of previous studies demonstrating an association between $A P O E$ genotype and cognitive reduction in TBI populations $(8,32,46,47)$. Conversely, other studies concluded that APOE- $\varepsilon 4$ carriers with TBI performed at a cognitive level similar to that of APOE- 4 non-carriers $(31,48-$ 50 ). These discrepancies might be attributable to differences in study design and methodologies applied. Our sample size was comparable to that of many previous studies on APOE- 44 and TBI in systematic reviews (32) and larger than the sample size in the study by Yue et al. (7).

Though more research is needed, our results suggest that APOE- $\varepsilon 4$ increased the likelihood of cognitive dysfunction the first months after trauma even in patients with mild TBIs. Although speculative, it is possible that possession of one copy of the APOE- $\varepsilon 4$ allele might result in increased risk of cognitive decline due to altered repair mechanisms following neurotrauma or exacerbate underlying MTBI-related cognitive impairment. The precise mechanisms responsible for the negative effects of the APOE- $\varepsilon 4$ allele on cognitive functioning following MTBI are not well-established. However, research supports the notion that, compared with the APOE $\varepsilon 2$ and $\varepsilon 3$ alleles, the $\varepsilon 4$ allele of the $A P O E$ gene possesses a number of properties that may hinder recovery following neurological injury (51). For example, APOE$\varepsilon 4$ genotype status was associated with increased cerebral edema and brain inflammation (52), which might result in slower brain recovery that in turn negatively influences cognitive performance early after MTBI. Individuals from a Norwegian population (53) have a lower frequency of APOE4 (APOE4 allele frequency of about $20 \%$ ) than in our study (37\%). It is possible that having the allele could represent a risk factor for suffering mTBI but this has to be explored in futures studies.

As MTBI varies greatly in clinical presentation, the shift to objective findings rather than subjective symptom complaints may offer a new approach for clinicians to understand patients' needs early after injury and determine when treatment changes are required (e.g., prevention of memory decline). Thus, finding a predictable marker of worse cognitive outcome would offer advantages in assessment of prognosis, allowing resources to be focused on the immediate aftermath of injury in those patients deemed vulnerable.

This study also investigated the occurrence of subjective cognitive and affective symptoms in MTBI patients at two months after injury. Everyday executive function complaints (BRIEF-A) and cognitive and emotional symptoms (RPQ) were more commonly reported by the APOE- $\varepsilon 4(+)$ carriers compared 
to non-carriers, which may suggest that subjective cognitive symptoms are a reflection of the immediate memory recall deficits. However, no significant between-group differences were found in ratings of depressive and anxiety symptoms (HADS) or functional outcome (GOSE) at two months post-injury. Our data suggest that there may be ways to both identify the neurocognitive and behavioral characteristics associated with MTBI and use APOE- $\varepsilon 4$ status to better manage the MTBI in APOE- $\varepsilon 4$ carriers. To our knowledge, these everyday executive changes reported by the APOE- $\varepsilon 4(+)$ carriers early after injury are novel findings in the MTBI literature.

With respect to intracranial injuries, the results showed a significantly higher proportion of epidural hematomas on acute $\mathrm{CT}$ in the group of APOE- $\varepsilon 4$ carriers. Although APOE- $\varepsilon 4$ carriers had a higher prevalence of epidural hematomas on acute $\mathrm{CT}$, there was not a higher prevalence of contusions or other intracranial injuries. This is line with the findings of Liaquat et al., who showed that the APOE genotype did not affect the risk of suffering an intracranial hematoma after head injury (26). Epidural hematomas occur more frequently among younger men (54), and although the difference was not significant, the mean age of the APOE- $\varepsilon 4(+)$ group was 3.7 years younger than that of the APOE- $\varepsilon 4(-)$ group. However, we cannot rule out that the significantly higher proportion of epidural hematomas on acute $\mathrm{CT}$ in the group of APOE- 44 carriers represents a Type 1 error from the relatively small sample size.

The present study has several strengths, including that it had a sample of patients with MTBI with no significant differences in patients' characteristics between the APOE- $\varepsilon 4$ carriers and noncarriers, as well as a prospective design. To our knowledge, this study is the first to combine data on a reliable memory test, subjective cognitive complaints, depressive symptoms, global functioning, and CT/MRI brain scans in groups of MTBI patients divided by APOE genotype. The relatively homogenous nature of our sample provides an advantageous setting in which to examine the relationship between the APOE genotype and neurocognitive functioning in patients with MTBI. The data presented here suggest that memory and everyday executive problems should be addressed early in MTBI patients with the APOE- $\varepsilon 4$ allele. However, this study has some limitations. Our findings are not generalizable to children, adolescents, and older adults or to patients with premorbid psychiatric history and drug abuse, as these patient groups were excluded from this study. There were no blast injuries included. Moreover, the number of participants included in our study is smaller than the recommended sample size for a genetic study (55). On the other hand, our sample size was large enough to detect medium effects sizes with acceptable power but a larger sample would be needed to have statistical power to detect small effect sizes. Therefore, this study might be underpowered to detect genetic differences in neurocognitive outcomes. A further limitation is the lack of a control group without TBI for comparison. Thus, we cannot rule out that the presence of APOE- $\varepsilon 4$ is associated with decreased verbal memory independent of a mTBI. Another limitation includes not assessing the patients' cognitive recovery over time, particularly when undertaking cognitive screening in APOE- $\varepsilon 4(+)$ carriers.
These patients may have significantly improved over time as described in a previous TBI study (56) and/or shown poorer outcome $(17,18)$. Finally, additional larger studies are required to determine if there is correlates of brain injury location with PCS symptoms or neuropsychological scores amongst APOE- $\varepsilon 4(+)$ carriers. Parenchymal contusions or axonal injuries to regions of eloquence may also impact outcome and represent an area of important and imminent future research.

\section{CONCLUSIONS}

Our findings suggest that carrying the APOE- 44 genotype has a negative effect on verbal memory two months after MTBI. Future investigations would benefit from larger sample sizes to increase statistical power as well as from using a longitudinal design. More research would be needed to assess the potential moderating influence of other genes implicated in neural recovery or protection. More detailed investigations into the pathophysiological consequences related to MTBI biomarkers may yield further insights and more useful MTBI biomarkers for clinical practice.

\section{DATA AVAILABILITY STATEMENT}

The datasets presented in this study can be found in online repositories. The names of the repository/repositories and accession number(s) can be found below: https://www.ncbi.nlm. nih.gov/genbank/, NM_000041.

\section{ETHICS STATEMENT}

The studies involving human participants were reviewed and approved by the Norwegian Regional Committee for Medical Research Ethics (2010/1899) (Oslo, Norway). The patients/participants provided their written informed consent to participate in this study. Written informed consent was obtained from the individual(s) for the publication of any potentially identifiable images or data included in this article.

\section{AUTHOR CONTRIBUTIONS}

TH, NA, and SS: conceptualization, drafting of the manuscript, and statistical analysis. TH, NA, ØH, EH, AS, KE, and SS: analysis, interpretation of data, and critical revision of the manuscript for important intellectual content. All authors have read and agreed to the published version of the manuscript.

\section{ACKNOWLEDGMENTS}

The authors thank all patients who participated in the study.

\section{SUPPLEMENTARY MATERIAL}

The Supplementary Material for this article can be found online at: https://www.frontiersin.org/articles/10.3389/fneur. 2022.735206/full\#supplementary-material 


\section{REFERENCES}

1. Rabinowitz AR, Li X, McCauley SR, Wilde EA, Barnes A, Hanten G, et al. Prevalence and predictors of poor recovery from mild traumatic brain injury. J Neurotrauma. (2015) 32:1488-96. doi: 10.1089/neu.2014.3555

2. Belanger HG, Curtiss G, Demery JA, Lebowitz BK, Vanderploeg RD. Factors moderating neuropsychological outcomes following mild traumatic brain injury: a meta-analysis. J Int Neuropsychol Soc. (2005) 11:215-27. doi: $10.1017 /$ S1355617705050277

3. Drag LL, Spencer RJ, Walker SJ, Pangilinan PH, Bieliauskas LA. The contributions of self-reported injury characteristics and psychiatric symptoms to cognitive functioning in OEF/OIF veterans with mild traumatic brain injury. J Int Neuropsychol Soc. (2012) 18:576-84. doi: $10.1017 /$ S1355617712000203

4. Ponsford J. Factors contributing to outcome following traumatic brain injury. NeuroRehabilitation. (2013) 32:803-15. doi: 10.3233/NRE-130904

5. Ponsford J, Willmott C, Rothwell A, Cameron P, Kelly AM, Nelms R, et al. Factors influencing outcome following mild traumatic brain injury in adults. J Int Neuropsychol Soc. (2000) 6:568-79. doi: 10.1017/S1355617700655066

6. McCauley SR, Wilde EA, Miller ER, Frisby ML, Garza HM, Varghese $\mathrm{R}$, et al. Preinjury resilience and mood as predictors of early outcome following mild traumatic brain injury. J Neurotrauma. (2013) 30:642-52. doi: $10.1089 /$ neu. 2012.2393

7. Yue JK, Robinson CK, Burke JF, Winkler EA, Deng H, Cnossen MC, et al. Apolipoprotein E epsilon 4 (APOE- $\varepsilon 4$ ) genotype is associated with decreased 6-month verbal memory performance after mild traumatic brain injury. Brain Behav. (2017) 7:e00791. doi: 10.1002/brb3.791

8. Merritt VC, Clark AL, Sorg SF, Evangelista ND, Werhane ML, Bondi MW, et al. Apolipoprotein E (APOE) epsilon4 genotype is associated with reduced neuropsychological performance in military veterans with a history of mild traumatic brain injury. J Clin Exp Neuropsychol. (2018) 40:1050-61. doi: 10.1080/13803395.2018.1508555

9. Liberman JN, Stewart WF, Wesnes K, Troncoso J. Apolipoprotein E epsilon 4 and short-term recovery from predominantly mild brain injury. Neurology. (2002) 58:1038-44. doi: 10.1212/WNL.58.7.1038

10. Sundström A, Marklund P, Nilsson LG, Cruts M, Adolfsson R, Van Broeckhoven C, et al. APOE influences on neuropsychological function after mild head injury: within-person comparisons. Neurology. (2004) 62:1963-6. doi: 10.1212/01.WNL.0000129268.83927.A8

11. Sundström A, Nilsson LG, Cruts M, Adolfsson R, Van Broeckhoven C, Nyberg L. Fatigue before and after mild traumatic brain injury: pre-post-injury comparisons in relation to Apolipoprotein E. Brain Inj. (2007) 21:1049-54. doi: 10.1080/02699050701630367

12. Bigler ED, Maxwell WL. Neuropathology of mild traumatic brain injury: relationship to neuroimaging findings. Brain Imaging Behav. (2012) 6:108-36. doi: 10.1007/s11682-011-9145-0

13. Eierud C, Craddock RC, Fletcher S, Aulakh M, King-Casas B, Kuehl D, et al. Neuroimaging after mild traumatic brain injury: Review and metaanalysis. NeuroImage Clinical. (2014) 4:283-94. doi: 10.1016/j.nicl.2013. 12.009

14. Mayer AR, Bellgowan PS, Hanlon FM. Functional magnetic resonance imaging of mild traumatic brain injury. Neurosci Biobehav Rev. (2015) 49:818. doi: 10.1016/j.neubiorev.2014.11.016

15. Zhou W, Xu D, Peng X, Zhang Q, Jia J, Crutcher KA. Meta-analysis of APOE4 allele and outcome after traumatic brain injury. J Neurotrauma. (2008) 25:279-90. doi: 10.1089/neu.2007.0489

16. Zeng S, Jiang JX, Xu MH, Xu LS, Shen GJ, Zhang AQ, et al. Prognostic value of apolipoprotein $\mathrm{E}$ epsilon4 allele in patients with traumatic brain injury: a meta-analysis and meta-regression. Genet Test Mol Biomarkers. (2014) 18:202-10. doi: 10.1089/gtmb.2013.0421

17. McFadyen CA, Zeiler FA, Newcombe V, Synnot A, Steyerberg E, Gruen RL, et al. Apolipoprotein E4 polymorphism and outcomes from traumatic brain injury: a living systematic review and meta-analysis. J Neurotrauma. (2019) 38:1124-36. doi: 10.1089/neu.2018.6052

18. Ponsford J, McLaren A, Schönberger M, Burke R, Rudzki D, Olver J, et al. The association between apolipoprotein $\mathrm{E}$ and traumatic brain injury severity and functional outcome in a rehabilitation sample. J Neurotrauma. (2011) 28:1683-92. doi: 10.1089/neu.2010.1623
19. Ost M, Nylén K, Csajbok L, Blennow K, Rosengren L, Nellgård B. Apolipoprotein E polymorphism and gender difference in outcome after severe traumatic brain injury. Acta Anaesthesiol Scand. (2008) 52:1364-9. doi: $10.1111 / j .1399-6576.2008 .01675 . x$

20. Wooten T, Sullivan DR, Logue MW, Fonda JR, Fortier CB, DeGutis J, et al. Apolipoprotein E (APOE) $\varepsilon 4$ status moderates the relationship between closerange blast exposure and cognitive functioning. J Int Neuropsychol Soc. (2021) 27:315-28. doi: 10.1017/S1355617720001034

21. Mahley RW, Nathan BP, Pitas RE. Apolipoprotein E. Structure, function, and possible roles in Alzheimer's disease. Ann N Y Acad Sci. (1996) 777:139-45. doi: 10.1111/j.1749-6632.1996.tb34412.x

22. Mahley RW, Weisgraber KH, Huang Y. Apolipoprotein E4: a causative factor and therapeutic target in neuropathology, including Alzheimer's disease. Proc Natl Acad Sci U S A. (2006) 103:5644-51. doi: 10.1073/pnas.0600549103

23. Horsburgh K, McCarron MO, White F, Nicoll JA. The role of apolipoprotein $\mathrm{E}$ in Alzheimer's disease, acute brain injury and cerebrovascular disease: evidence of common mechanisms and utility of animal models. Neurobiol Aging. (2000) 21:245-55. doi: 10.1016/S0197-4580(00)00097-X

24. Shi Y, Yamada K, Liddelow SA, Smith ST, Zhao L, Luo W, et al. ApoE4 markedly exacerbates tau-mediated neurodegeneration in a mouse model of tauopathy. Nature. (2017) 549:523-7. doi: 10.1038/nature24016

25. Smith C, Graham DI, Murray LS, Stewart J, Nicoll JA. Association of APOE e4 and cerebrovascular pathology in traumatic brain injury. J Neurol Neurosurg Psychiatry. (2006) 77:363-6. doi: 10.1136/jnnp.2005.074617

26. Liaquat I, Dunn LT, Nicoll JA, Teasdale GM, Norrie JD. Effect of apolipoprotein E genotype on hematoma volume after trauma. J Neurosurg. (2002) 96:90-6. doi: 10.3171/jns.2002.96.1.0090

27. Leclercq PD, Murray LS, Smith C, Graham DI, Nicoll JA, Gentleman SM. Cerebral amyloid angiopathy in traumatic brain injury: association with apolipoprotein E genotype. J Neurol Neurosurg Psychiatry. (2005) 76:229-33. doi: 10.1136/jnnp.2003.025528

28. Merritt VC, Arnett PA. Apolipoprotein E (APOE) $\epsilon 4$ allele is associated with increased symptom reporting following sports concussion. J Int Neuropsychol Soc. (2016) 22:89-94. doi: 10.1017/S1355617715001022

29. Banks SJ, Miller JB, Rissman RA, Bernick CB. Lack of influence of apolipoprotein e status on cognition or brain structure in professional fighters. J Neurotrauma. (2017) 34:380-4. doi: 10.1089/neu.2016.4453

30. Merritt VC, Rabinowitz AR, Arnett PA. The influence of the Apolipoprotein E (APOE) gene on subacute post-concussion neurocognitive performance in college athletes. Arch Clin Neuropsychol. (2018) 33:36-46. doi: 10.1093/arclin/acx051

31. Padgett CR, Summers MJ, Skilbeck CE. Is APOE $\varepsilon 4$ associated with poorer cognitive outcome following traumatic brain injury? A meta-analysis. Neuropsychology. (2016) 30:775-90. doi: 10.1037/neu0000270

32. Lawrence DW, Comper P, Hutchison MG, Sharma B. The role of apolipoprotein E episilon $(\varepsilon)-4$ allele on outcome following traumatic brain injury: a systematic review. Brain injury. (2015) 29:1018-31. doi: 10.3109/02699052.2015.1005131

33. Head Injury Interdisciplinary Special Interest Group of the American Congress of Rehabilitation. Definition of mild traumatic brain injury. J Head Trauma Rehabil. (1993) 8:86-7. doi: 10.1097/00001199-199309000-00010

34. Untergasser A, Cutcutache I, Koressaar T, Ye J, Faircloth BC, Remm M, et al. Primer3-new capabilities and interfaces. Nucleic Acids Res. (2012) 40:e115. doi: 10.1093/nar/gks596

35. Holthe OO, Hellstrom T, Andelic N, Server A, Sigurdardottir S. Improvement and prediction of memory and executive functions in patients admitted to a neurosurgery service with complicated and uncomplicated mild traumatic brain injury. J Head Trauma Rehabil. (2019) 34:E45-e56. doi: 10.1097/HTR.0000000000000463

36. Teasdale G, Jennett B. Assessment of coma and impaired consciousness. A practical scale. Lancet. (1974) 2:81-4. doi: 10.1016/S0140-6736(74)91639-0

37. Delis DC, Kramer JH, Kaplan E, Ober BA. California Verbal Learning Test-Second Edition. Adult Version Manual. San Antonio, TX: Psychological Corporation (2000).

38. Woods SP, Delis DC, Scott JC, Kramer JH, Holdnack JA. The California verbal learning test-second edition: test-retest reliability, practice effects, and reliable change indices for the standard and alternate forms. Arch Clin Neuropsychol. (2006) 21:413-20. doi: 10.1016/j.acn.2006.06.002 
39. Roth RM, Isquith PK, Gioia GA. Behavior Rating Inventory of Executive Functions-Adult Version (Brief A). Professional Manual. Psychological Assessment Resources, Inc (PAR) (2005).

40. King NS, Crawford S, Wenden FJ, Moss NE, Wade DT. The rivermead post concussion symptoms questionnaire: a measure of symptoms commonly experienced after head injury and its reliability. J Neurol. (1995) 242:587-92. doi: $10.1007 / \mathrm{BF} 00868811$

41. Zigmond AS, Snaith RP. The hospital anxiety and depression scale. Acta Psychiatr Scand. (1983) 67:361-70. doi: 10.1111/j.1600-0447.1983.tb09716.x

42. Schonberger M, Ponsford J. The factor structure of the hospital anxiety and depression scale in individuals with traumatic brain injury. Psychiatry Res. (2010) 179:342-9. doi: 10.1016/j.psychres.2009.07.003

43. Whelan-Goodinson R, Ponsford J, Schonberger M. Validity of the hospital anxiety and depression scale to assess depression and anxiety following traumatic brain injury as compared with the structured clinical interview for DSM-IV. J Affect Disord. (2009) 114:94-102. doi: 10.1016/j.jad.2008.06.007

44. Wilson JT, Pettigrew LE, Teasdale GM. Structured interviews for the Glasgow outcome scale and the extended Glasgow outcome scale: guidelines for their use. J Neurotrauma. (1998) 15:573-85. doi: 10.1089/neu.1998. 15.573

45. Faul F, Erdfelder E, Lang AG, Buchner A. G*Power 3: a flexible statistical power analysis program for the social, behavioral, and biomedical sciences. Behav Res Methods. (2007) 39:175-91. doi: 10.3758/BF031 93146

46. Ariza M, Pueyo R, Matarín Mdel M, Junqué C, Mataró M, Clemente I, et al. Influence of APOE polymorphism on cognitive and behavioural outcome in moderate and severe traumatic brain injury. J Neurol Neurosurg Psychiatry. (2006) 77:1191-3. doi: 10.1136/jnnp.2005.085167

47. Eramudugolla R, Bielak AA, Bunce D, Easteal S, Cherbuin N, Anstey KJ. Long-term cognitive correlates of traumatic brain injury across adulthood and interactions with APOE genotype, sex, and age cohorts. Journal of the International Neuropsychological Society. (2014) 20:444-54. doi: 10.1017/S1355617714000174

48. Pruthi N, Chandramouli BA, Kuttappa TB, Rao SL, Subbakrishna DK, Abraham MP, et al. Apolipoprotein E polymorphism and outcome after mild to moderate traumatic brain injury: a study of patient population in India. Neurol India. (2010) 58:264-9. doi: 10.4103/0028-3886. 63810

49. Shadli RM, Pieter MS, Yaacob MJ, Rashid FA. APOE genotype and neuropsychological outcome in mild-to-moderate traumatic brain injury: a pilot study. Brain injury. (2011) 25:596-603. doi: $10.3109 / 02699052.2011 .572947$
50. Ponsford J, Rudzki D, Bailey K, Ng KT. Impact of apolipoprotein gene on cognitive impairment and recovery after traumatic brain injury. Neurology. (2007) 68:619-20. doi: 10.1212/01.wnl.0000254609.04330.9d

51. Samatovicz RA. Genetics and brain injury: apolipoprotein E. J Head Trauma Rehabil. (2000) 15:869-74. doi: 10.1097/00001199-200006000-00002

52. Lynch JR, Pineda JA, Morgan D, Zhang L, Warner DS, Benveniste H, et al. Apolipoprotein $\mathrm{E}$ affects the central nervous system response to injury and the development of cerebral edema. Ann Neurol. (2002) 51:113-7. doi: 10.1002/ana.10098

53. Kumar T, Liestøl K, Maehlen J, Hiorth A, Jettestuen E, Lind H, et al. Allele frequencies of apolipoprotein $\mathrm{E}$ gene polymorphisms in the protein coding region and promoter region $(-491 \mathrm{~A} / \mathrm{T})$ in a healthy Norwegian population. Hum Biol. (2002) 74:137-42. doi: 10.1353/hub.2002.0006

54. Aromatario M, Torsello A, D’Errico S, Bertozzi G, Sessa F, Cipolloni L, et al. Traumatic epidural and subdural hematoma: epidemiology, outcome, and dating. Medicina (Kaunas, Lithuania). (2021) 57:125. doi: $10.3390 /$ medicina57020125

55. Hong EP, Park JW. Sample size and statistical power calculation in genetic association studies. Genomics Inform. (2012) 10:117-22. doi: $10.5808 /$ GI.2012.10.2.117

56. Noé E, Ferri J, Colomer C, Moliner B, Chirivella J. APOE genotype and verbal memory recovery during and after emergence from post-traumatic amnesia. Brain Inj. (2010) 24:886-92. doi: 10.3109/02699051003724952

Conflict of Interest: The authors declare that the research was conducted in the absence of any commercial or financial relationships that could be construed as a potential conflict of interest.

Publisher's Note: All claims expressed in this article are solely those of the authors and do not necessarily represent those of their affiliated organizations, or those of the publisher, the editors and the reviewers. Any product that may be evaluated in this article, or claim that may be made by its manufacturer, is not guaranteed or endorsed by the publisher.

Copyright (c) 2022 Hellstrøm, Andelic, Holthe, Helseth, Server, Eiklid and Sigurdardottir. This is an open-access article distributed under the terms of the Creative Commons Attribution License (CC BY). The use, distribution or reproduction in other forums is permitted, provided the original author(s) and the copyright owner(s) are credited and that the original publication in this journal is cited, in accordance with accepted academic practice. No use, distribution or reproduction is permitted which does not comply with these terms. 